Research Article

\title{
Comprehensive Analysis of Differently Expressed and Methylated Genes in Preeclampsia
}

\author{
Wenyi Xu, ${ }^{1}$ Ping Ru, ${ }^{1}$ Zhuorong Gu, ${ }^{1}$ Ruoxi Zhang, ${ }^{1}$ Xixia Pang, ${ }^{2}$ Yi Huang, ${ }^{3}$ Zhou Liu $\mathbb{D},{ }^{4}$ \\ and Ming Liu \\ ${ }^{1}$ Department of Obstetrics and Gynecology, Tongren Hospital, Shanghai Jiao Tong University School of Medicine, \\ Shanghai 200032, China \\ ${ }^{2}$ Department of Obstetrics and Gynecology, Kongjiang Hospital, Shanghai 200093, China \\ ${ }^{3}$ Department of Life Science, Sichuan Agricultural University, Sichuan 625014, China \\ ${ }^{4}$ Department of Health Sciences Affiliated Zhoupu Hospital, Shanghai University of Medicine, Shanghai 200032, China
}

Correspondence should be addressed to Zhou Liu; zpyyfck@126.com and Ming Liu; ming_12016@126.com

Received 23 June 2020; Revised 18 August 2020; Accepted 6 September 2020; Published 2 November 2020

Academic Editor: Lei Chen

Copyright (c) 2020 Wenyi Xu et al. This is an open access article distributed under the Creative Commons Attribution License, which permits unrestricted use, distribution, and reproduction in any medium, provided the original work is properly cited.

Preeclampsia (PE) is one of the mainly caused maternal and infant incidences and mortalities worldwide. However, the mechanisms underlying PE remained largely unclear. The present study identified 1716 high expressions of gene and 2705 low expressions of gene using GSE60438 database, and identified 7087 hypermethylated and 15120 hypomethylated genes in preeclampsia using GSE100197. Finally, 536 upregulated genes with hypomethylation and 322 downregulated genes with hypermethylation were for the first time revealed in PE. Gene Ontology (GO) analysis revealed that these genes were associated with peptidyl-tyrosine phosphorylation, skeletal system development, leukocyte migration, transcription regulation, $\mathrm{T}$ cell receptor and IFN- $\boldsymbol{\gamma}$-involved pathways, innate immune response, signal transduction, cell adhesion, angiogenesis, and hemopoiesis. Kyoto Encyclopedia of Genes and Genomes (KEGG) pathway analysis demonstrated that aberrantly methylated differentially expressed genes were involved in regulating adherens junction, pluripotency of stem cell regulation, immune processing, T cell receptor and NF- $\kappa$ B pathways, HTLV-I and HSV infections, leishmaniasis, and NK-induced cytotoxicity. Protein-protein interaction (PPI) network analysis identified several hub networks and key genes, including MAPK8, CCNF, CDC23, ABL1, NF1, UBE2E3, CD44, and PIK3R1. We hope these findings will draw more attention to these hub genes in future PE studies.

\section{Background}

As a kind of pregnancy-induced hypertension, preeclampsia $(\mathrm{PE})$ is one of the mainly caused maternal and infant incidences and mortalities worldwide $[1,2]$. Numerous body organs and functional systems could be affected by PE, followed by emerging renal failure, ischemic heart, type II diabetes, etc. [1-3]. Several researches have shown a part of external and internal factors that had been identified to induce PE [4]. Currently, trophoblast invasion and failure of spiral artery transformation have been considered to be one inducer of PE [5]. Even though perinatal care was improved, the ratio occurrence of PE has not been reduced
$[6,7]$. Up to date, the inherent mechanism of PE taken part in many physiological disorders stayed elusive.

Many studies have identified a large number of differentially expressed genes (DEGs) and differentially methylated genes (DMGs) in PE based on advanced technologies [812]. Liu et al. reported that 268 dysfunctional genes were identified in $\mathrm{PE}$, which were related to hormone activity and immune response. Besides, this study revealed TLR2, GSTO1, and mapk13 functioned importantly in the progression of PE $[10,11]$. Presently, no studies to investigate the regulated role of gene expression implicated in $\mathrm{PE}$.

Epigenetics indicated that the change of gene expression was heritable, but did not turn out to be in DNA $[13,14]$. 
Among them, DNA methylation was the mostly generated modification in biological metabolism [15]. DNA methyltransferases (DNMTs) were responsible for transmitting DNA methylation to target sites [16]. Nevertheless, the details towards the methylation are not fully understood.

Here, we wanted to explore the association of gene expression with DNA methylation and potential signal pathway in PE development. Therefore, we evaluated the unknown interaction and related signaling pathways of DEG and DMGs in PE by gene expression microarray data (GSE60438) [12] and gene methylation microarray data (GSE100197) [17]. To this end, we attempted to uncover the potential indicator for early diagnosis and prognosis of $\mathrm{PE}$, and also give a hint of probing the involved pathways of DEG/DMGs in PE.

\section{Materials and Methods}

2.1. Microarray Data. Differently expressed genes (DEGs)/differently methylated genes (DMGs) were individually analyzed by GSE60438 [12] (including 47 preeclampsia and 48 normal samples) and GSE100197 (including 22 preeclampsia and 51 normal samples) [17]. The details could be seen in the website https://www.ncbi.nlm.nih.gov/geo/.

2.2. Data Processing. GEO2R is an online tool that allows users to perform comparisons between different groups in GEO series, which depends on the GEOquery and the Linear Models for Microarray Analysis (LIMMA) R packages [18, 19]. The raw data in TXT format were checked in Venn software online to detect the commonly DEGs among the three datasets. The cutoff standards of DEGs were defined as $P<$ 0.05 and fold change $>2$, while those of DMGs were indicated as FDR $<0.05$ and a fold change $>2$.

2.3. The Gene Ontology (GO) and Kyoto Encyclopedia of Genes and Genomes (KEGG) Pathway Analysis. DAVID [20] was conducted to do bioinformatics analysis. Significant difference was indicated as $P<0.01$.

2.4. Construction of Protein-Protein Interaction (PPI) Network. PPI network, including highly methylated and lowly methylated genes, was constructed by STRING database. Interaction score of 0.4 was regarded as cutoff. Cytoscape and the Molecular Complex Detection (MCODE) algorithm were separately applied to visualize PPI network and screen modules. The Molecular Complex Detection (MCODE) app was used to analyze PPI network modules [21], and MCODE scores $>3$ and the number of nodes $>5$ were set as cutoff criteria with the default parameters (degree cutoff $\geq 2$, node score cutoff $\geq 2, \quad K$-core $\geq 2$, and max depth $=100$ ). DAVID was utilized to perform pathway enrichment analysis of gene modules. Finally, cytoHubba, a Cytoscape plugin, was utilized to explore PPI network hub genes; it provides a user-friendly interface to explore important nodes in biological networks and computes using eleven methods, of which MCC has a better performance in the PPI network [22].

\section{Results}

3.1. Identification of Aberrantly Methylated DEGs in PE. After microarray analysis, our data have shown upregulated and downregulated 3378 DEGs which were 1663 and 1715, respectively. We identified 7087 highly methylated and 15120 lowly methylated genes in PE after relative to normal samples. 829 highly methylated genes (Figure 1(c)) with enhanced level and 408 lowly methylated genes (Figure 1(d)) with weak level were classified after overlapping DEGs and aberrantly methylated genes. Figure 1(a) shows DEGs in GSE60438 and Figure 1(b) illustrates DMGs of PE and normal tissue. The top 10 upregulated and downregulated genes in PE are shown in Tables 1 and 2.

3.2. Functional Analysis. GO analysis indicated that high methylation of genes with increasing expression was generally concentrated in peptidyl-tyrosine phosphorylation, skeletal system development, regulation of bone resorption, mitotic cell cycle, peptidyl-serine phosphorylation pathway, movement of cell or subcellular component, axonogenesis, retina layer formation, calcium ion homeostasis, and cell proliferation (Figure 2(a)).

Low methylation of genes with reduced expression was abounded in leukocyte migration, transcription regulation, $\mathrm{T}$ cell receptor and IFN- $\gamma$-involved pathways, innate immune response, signal transduction, cell adhesion, angiogenesis, and hemopoiesis (Figure 2(b)).

3.3. Analysis of Pathway. Upregulated genes with high methylation were dramatically enriched in adherens junction, pluripotency of stem cell regulation, proteoglycans in cancer, the ErbB and sphingolipid signaling pathways, actin cytoskeleton process, ovarian steroidogenesis, carbon metabolism, renal carcinoma, and metabolic pathways (Figure 3(a)).

Downregulated genes with hypermethylation were enriched in cell adhesion, immune processing, $\mathrm{T}$ cell receptor and NF- $\kappa$ B pathways, HTLV-I and HSV infection, leishmaniasis, and NK-induced cytotoxicity (Figure 3(b)).

3.4. PPI Network Establishment and cytoHubba Analysis. For strong expression of genes with hypomethylation, 264 nodes and 456 edges were elected. For weak expression of genes with hypermethylation, 159 nodes and 290 edges were obtained (Figure 4). For upregulated oncogenes with hypomethylation, 380 nodes and 1170 edges are shown in Figures 4 and 5. Downregulated TSGs with hypermethylation are indicated in (Figure 5). Totally, 212 nodes and 458 edges were included in TSGs. MCODE plugin detection revealed that FLNA and PRKCB were reduced with hypermethylation, and AKT1, PRDM10, CCND1, and FASN 4 were heightened with hypomethylation.

3.5. Key Module and Gene Analysis. There is obvious difference between three modules with hypomethylation of upregulated genes and three modules with hypermethylation of downregulated genes (Figure 4). The hub network 1 of overexpressed hypomethylated genes included CCNF, RNF14, UBE2B, SH3RF1, UBE2V1, FBXO30, FBXW7, FBXO17, PJA2, UBE2M, TRIM36, HECW2, UBE2E3, SOCS1, MYLIP, 


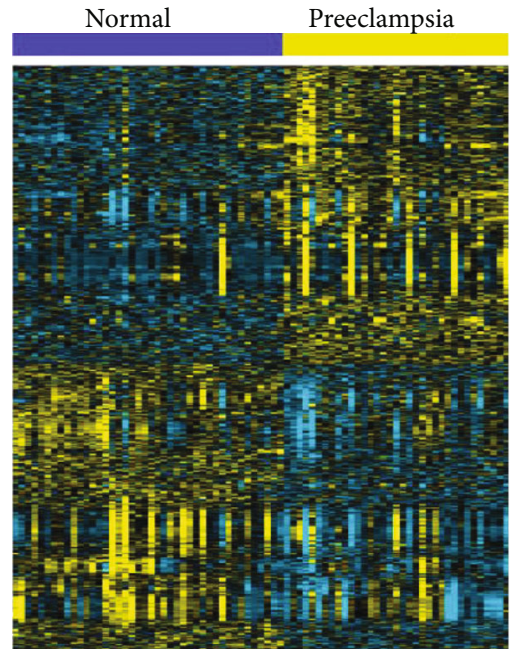

(a)

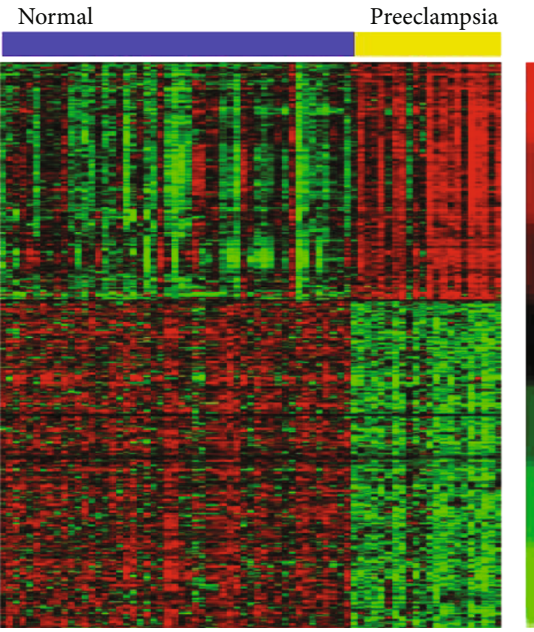

(b)

Down-regulated

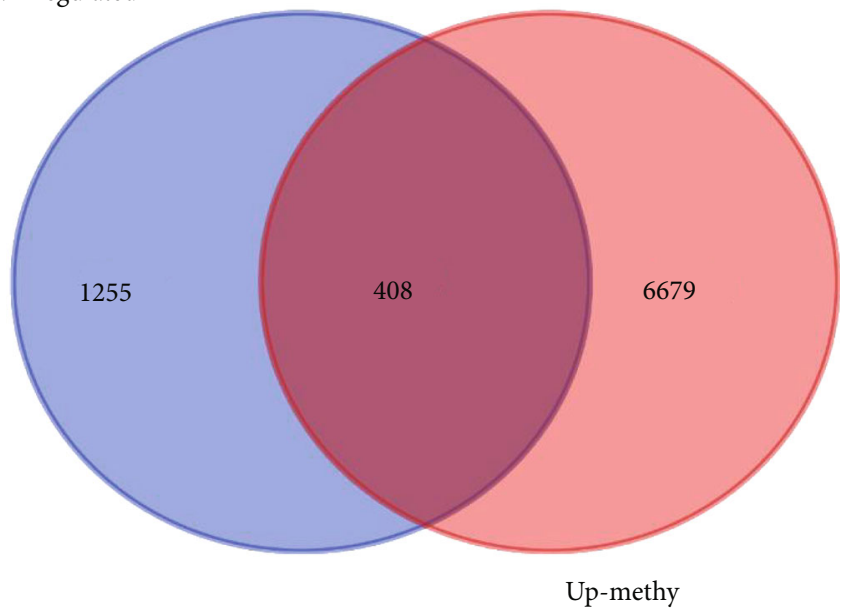

(c)

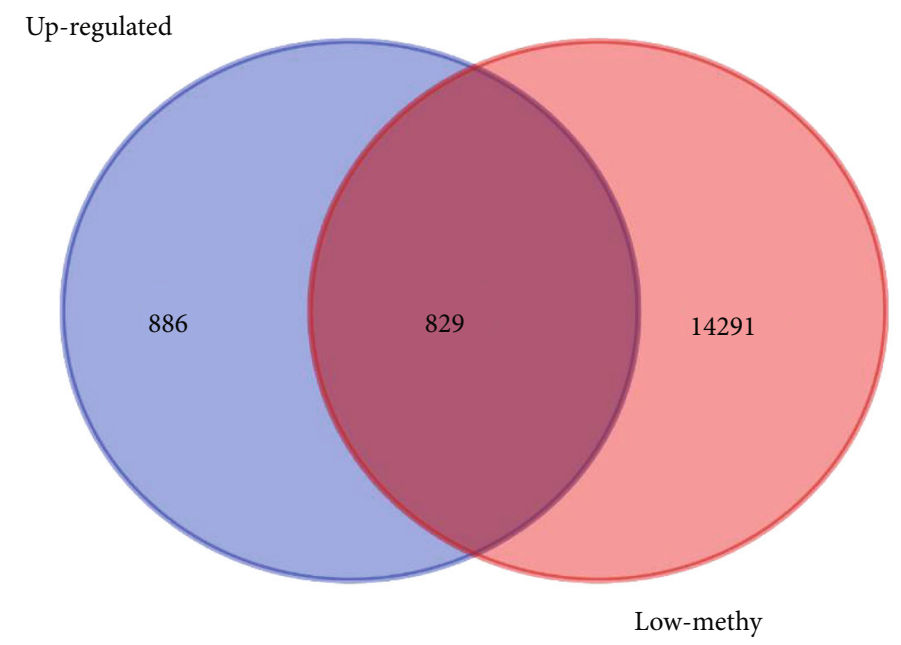

(d)

FIGURE 1: Identification of aberrantly methylated differentially expressed genes in PC. (a) Identification of differently expressed genes in PC using GSE60438. (b) Identification of differently methylated genes in PC using GSE100197. (c) A total of 829 upregulated hypomethylated genes were identified in PC. (d) A total of 409 downregulated hypermethylated genes were identified in PC. 
TABLE 1: The top 10 upregulated genes in PE compared to normal samples.

\begin{tabular}{lcccc}
\hline Gene & AVE NC & AVE PE & FC & $P$ value \\
\hline CGB5 & 7.140694905 & 8.555772571 & 2.666740913 & 0.000524413 \\
CRH & 7.35786819 & 8.743007971 & 2.611972633 & 0.00019934 \\
CGB1 & 7.330520262 & 8.540119829 & 2.312734358 & 0.00097466 \\
KISS1 & 7.882624452 & 9.119308971 & 2.356563438 & 0.00381749 \\
ADAM12 & 8.732927738 & 10.04238443 & 2.478481844 & 0.002319055 \\
DLK1 & 7.393902548 & 8.500453743 & 2.153302782 & 0.010929138 \\
CGA & 8.495501333 & 9.765370743 & 2.41139737 & 0.003638636 \\
PSG6 & 8.514054476 & 9.723809743 & 2.312983969 & 0.007442872 \\
CGB8 & 7.176896333 & 8.174694886 & 1.996950473 & 0.001320154 \\
PAGE4 & 7.430149214 & 8.450650829 & 2.028624174 & 0.009079707 \\
\hline
\end{tabular}

TABLE 2: The top 10 downregulated genes in PE compared to normal samples.

\begin{tabular}{lcccc}
\hline Gene & AVE NC & AVE PE & FC & $P$ value \\
\hline LOC647169 & 8.7158875 & 8.070096229 & 0.639142146 & 0.012472714 \\
FCN1 & 10.84868995 & 10.03952706 & 0.570712911 & 0.023728745 \\
LYZ & 12.23783017 & 11.29681926 & 0.520867776 & 0.002834118 \\
CCL2 & 10.03269452 & 9.2354408 & 0.575443535 & 0.000263349 \\
CX3CR1 & 8.787585643 & 8.068285229 & 0.607391905 & 0.007852246 \\
CCL18 & 8.994379333 & 8.217637057 & 0.583683311 & 0.000247839 \\
GSTA1 & 8.678950238 & 7.926187143 & 0.593465844 & 0.006579547 \\
PI3 & 8.461040238 & 7.713865943 & 0.595769307 & 0.013088748 \\
LTB & 9.728131619 & 8.857907943 & 0.547062027 & 0.00330664 \\
GSTA1 & 8.871995429 & 8.0231716 & 0.555237214 & 0.004279029 \\
\hline
\end{tabular}

and CDC23. The hub network 2 of overexpressed hypomethylated genes included GPER1, OPN4, GPR17, PLCB4, MCHR2, MCHR1, TAS2R14, PTGER3, CCL4, NPS, KISS1, and ADCY8. The hub network 3 of overexpressed hypomethylated genes included SEC22B, LHB, CGA, HNRNPA3, NEIL3, TAAR6, SLC30A5, GOLIM4, BAG4, ABCB1, GOLGA5, MAN1A2, CRH, PTPN6, PREB, SEC24B, FOLR1, DEPDC1B, TPX2, SLC30A2, CEP152, FGFR1, SGOL2, LIMK1, PSG3, CDC25C, KHSRP, DHX9, SYNCRIP, PAK4, ERBB2, SDC3, SDC1, PSG6, JUP, DCTN3, RPL22L1, KRT19, NUF2, PSG11, NCAPG, QPCT, RHOBTB1, RPL34, SRP19, YWHAE, MATR3, NTF3, LMAN1, PSG4, ERBB3, SPCS3, SEC11A, ARHGEF11, SLC30A1, SLC39A1, TROAP, MAN1C1, MAP2K1, RRAS2, AKT3, SLC39A8, PSG9, TRIP13, TIMP2, TRIM24, and PSG1.

The hub network 1 of downregulated hypermethylated genes included ATG7, UBA7, RNF213, ARIH2, FBXL19, FBXO44, HERC4, and ASB15. The hub network 2 of downregulated hypermethylated genes included SRSF4, RBM5, PRPF3, SF3B1, HNRNPU, CPSF2, and CSTF3. The hub network 3 of downregulated hypermethylated genes included ADCY7, ZAP70, GPR18, LY9, NPBWR1, CD4, ITGA4, CD44, FPR3, SSTR1, GABBR1, GNB4, CCR3, and SLAMF1 (Figure 5).

Among these genes, MAPK8, CCNF, CDC23, ABL1, NF1, UBE2E3, CD44, and PIK3R1 were identified as key reg- ulators in PE by connecting with more than 20 different genes in the network.

\section{Discussion}

Preeclampsia was reported to be largely related to increasing incidence and death of maternal organ, dysfunction of maternal organ, or restricted growth of foetal organ [23]. However, the mechanisms related to this disease remained largely unclear. Emerging studies demonstrated that the aberrant changes in DNA methylation contributed to the abnormal expression of key genes in multiple diseases, such as preeclampsia [24]. Therefore, conclusive delineation of gene level and methylation could provide novel insights to identify novel predictive and therapeutic targets for preeclampsia. The present study identified 1716 high expressions of gene and 2705 low expressions of gene using GSE60438 database, and identified 7087 hypermethylated and 15120 hypomethylated genes in preeclampsia using GSE100197 database. Finally, 536 upregulated genes with hypomethylation and 322 downregulated genes with hypermethylation were for the first time revealed in PE.

Furthermore, bioinformatics analysis was performed to reveal the potential functions of these aberrantly methylated DEGs in preeclampsia. Meanwhile, we identified aberrantly methylated DEGs in preeclampsia that were 


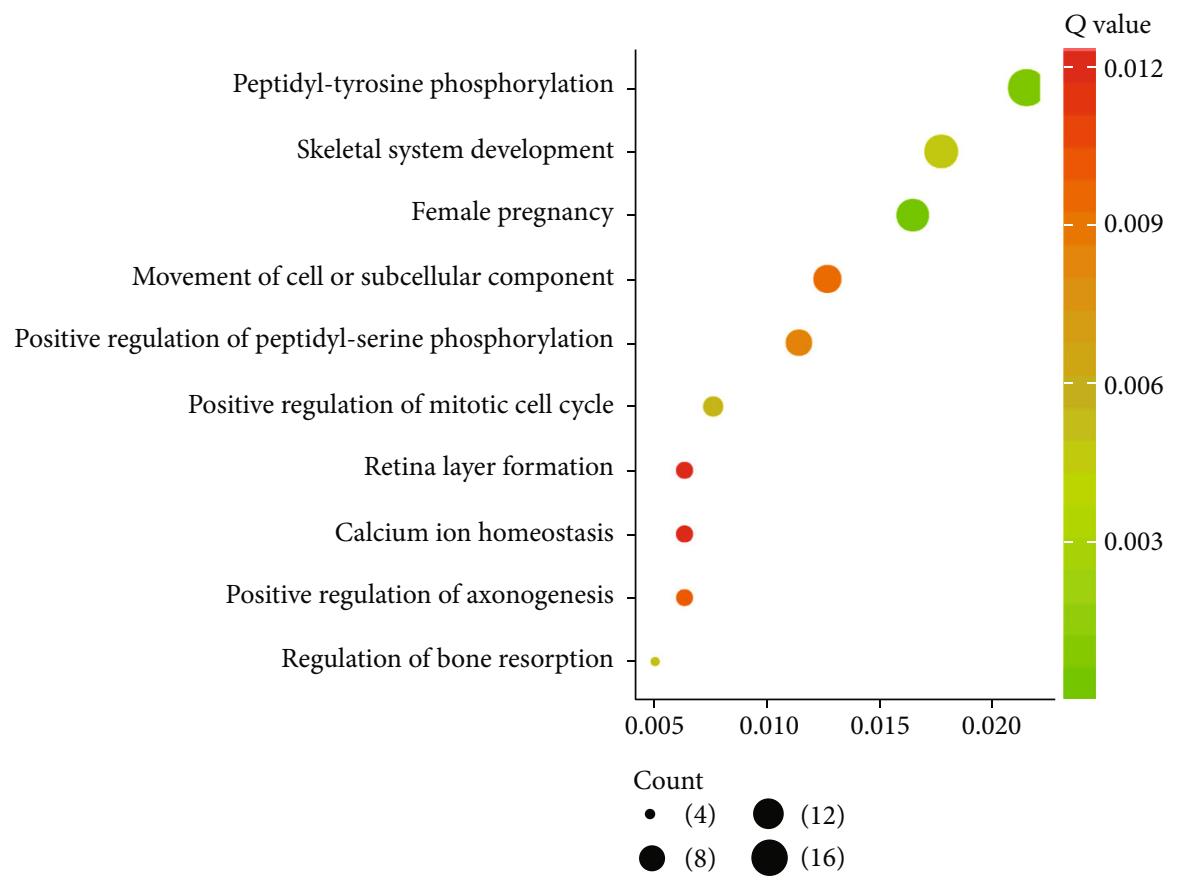

(a)

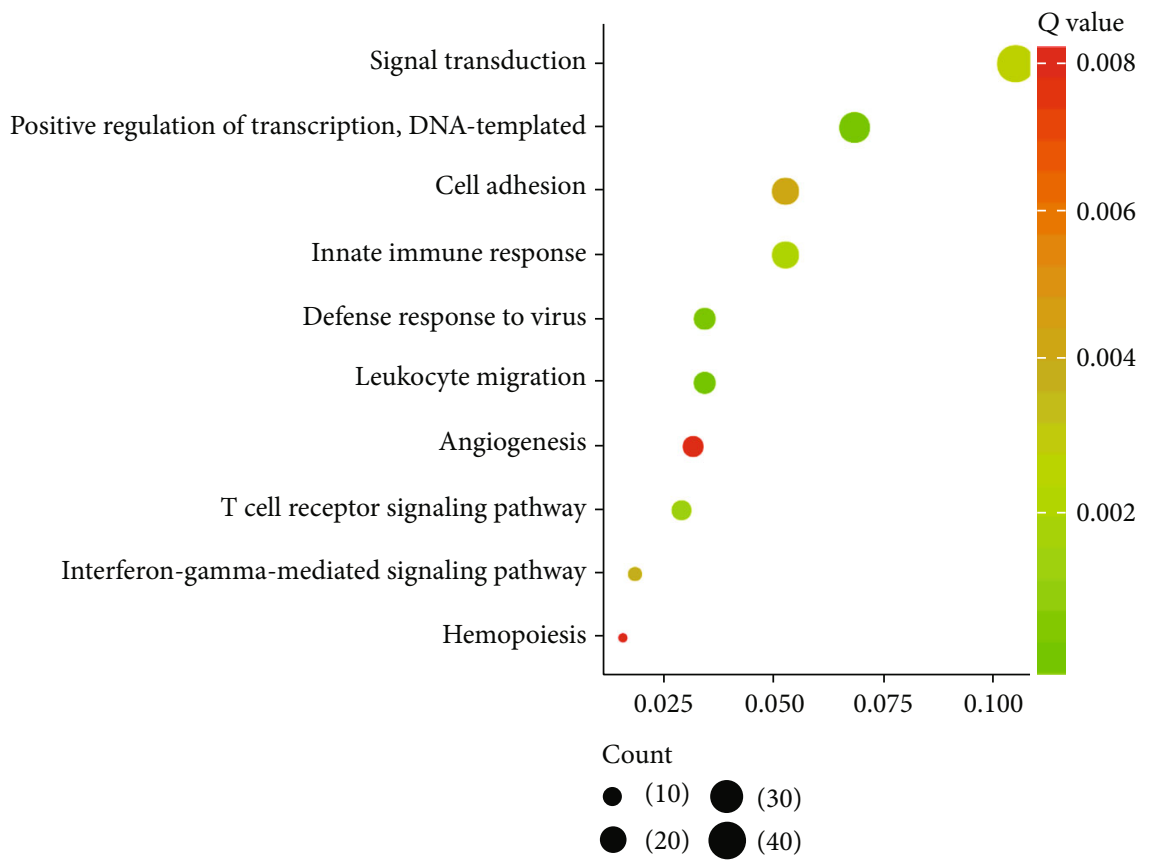

(b)

FIGURE 2: GO analysis of aberrantly methylated differentially expressed genes in PC. GO analysis of upregulated hypomethylated genes (a) and downregulated hypermethylated genes (b) in PC.

associated with transcription level, cell defense, cell immunity response, IFN- $\gamma$-involved pathway, and $\mathrm{T}$ cell receptor pathway. These findings were consistent with previous reports that abnormal regulation of immune functions was related to preeclampsia progression [25]. Our results showed that hypomethylated highly expressed genes were related to the regulation of multiple key signalings in cell biology, such as cell mitosis, axonogenesis, $\mathrm{Ca}^{2+}$ homeosta- sis, cell proliferation, the ErbB signaling pathway, ovarian steroidogenesis, and the sphingolipid signaling pathway. As a second messenger, $\mathrm{Ca}^{2+}$ acts as a primary role in cell growth, cell death, etc. [26]. Downstream pathway was activated by $\mathrm{Ca}^{2+}$ via exporting intracellular organelles or importing extracellular depots [27-29]. As the foremost form of $\mathrm{Ca}^{2+}$ pathway, downstream effectors of intracellular $\mathrm{Ca}^{2+}$ oscillations included transcription factors, kinases, and 


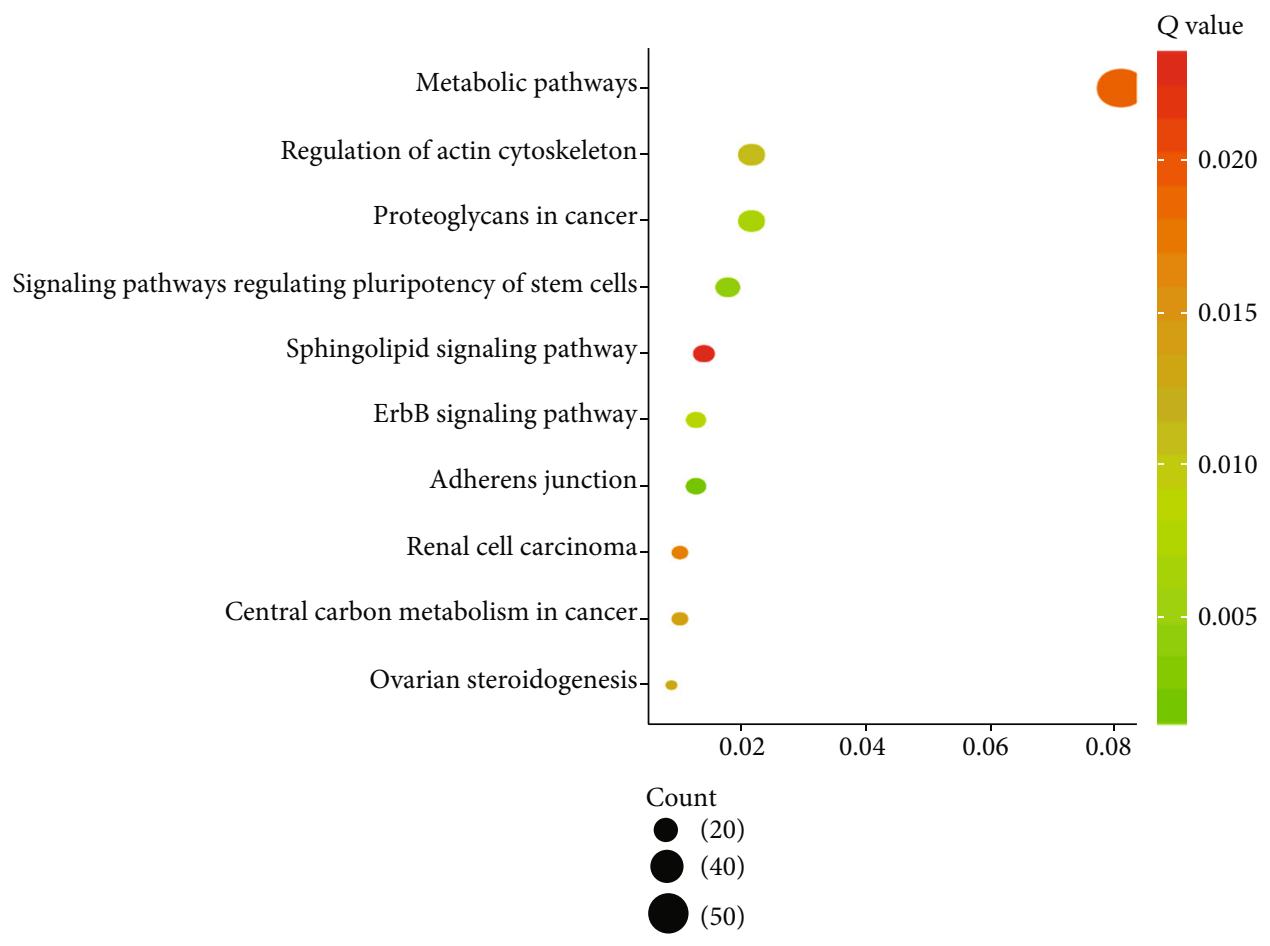

(a)

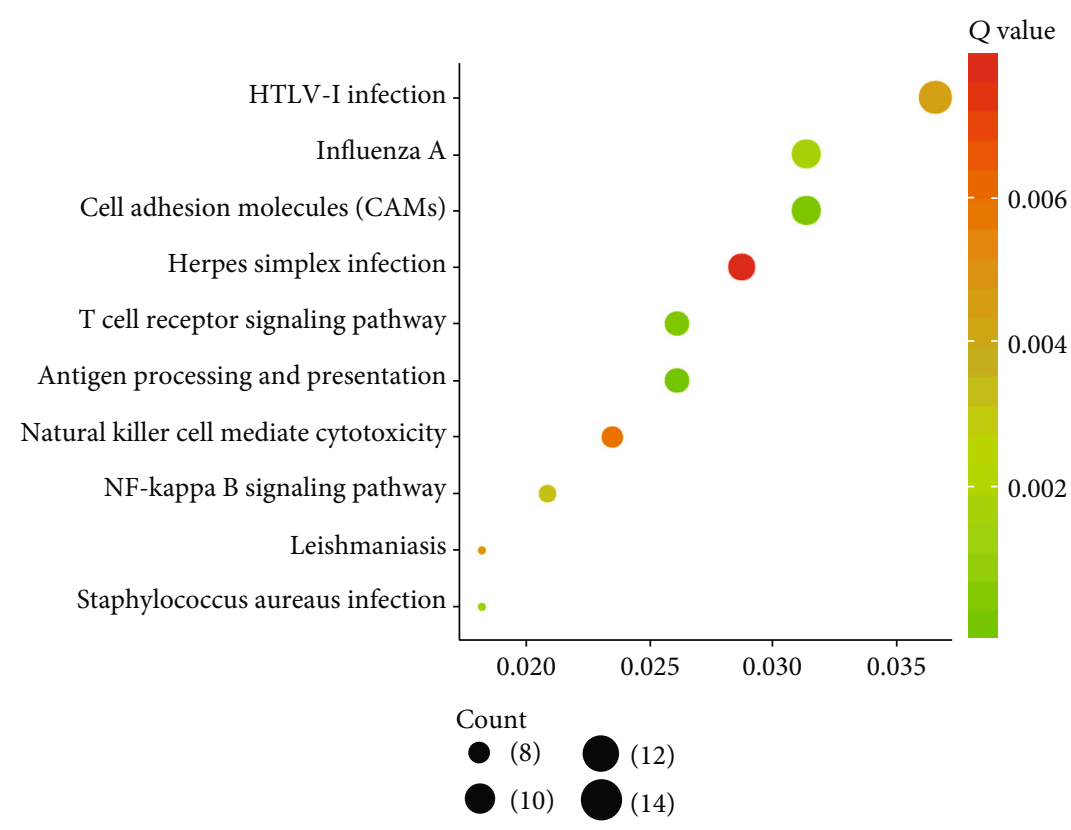

(b)

FIGURE 3: KEGG pathway analysis of aberrantly methylated differentially expressed genes in PC. KEGG pathway analysis of upregulated hypomethylated genes (a) and downregulated hypermethylated genes (b) in PC.

other functional proteins [30-32]. Our data suggests that the imbalance of $\mathrm{Ca}^{2+}$ in homeostatic cells may be linked to the progression of PE. A very interesting finding is that a recent study showed that $\mathrm{Ca}^{2+}$ signaling is related to the activation of the ErbB pathway, involving lots of tyrosine kinases, and is resistant to radiation and chemotherapy in many tumors. Two tyrosine residues were dimerized and phosphorylated by EGFR after conjugating to ligands [33,
34]. Conversely, these phosphorylated tyrosines could be regarded as binding sites for some signal transmitters which participated in biological pathways.

Moreover, we revealed that hypermethylated genes with low expression were associated with cell adhesion, angiogenesis, hemopoiesis, and the NF-kappa B signaling pathway. A recent study showed that the genes of cell adhesion signaling in the preeclamptic placentas were observed to be 


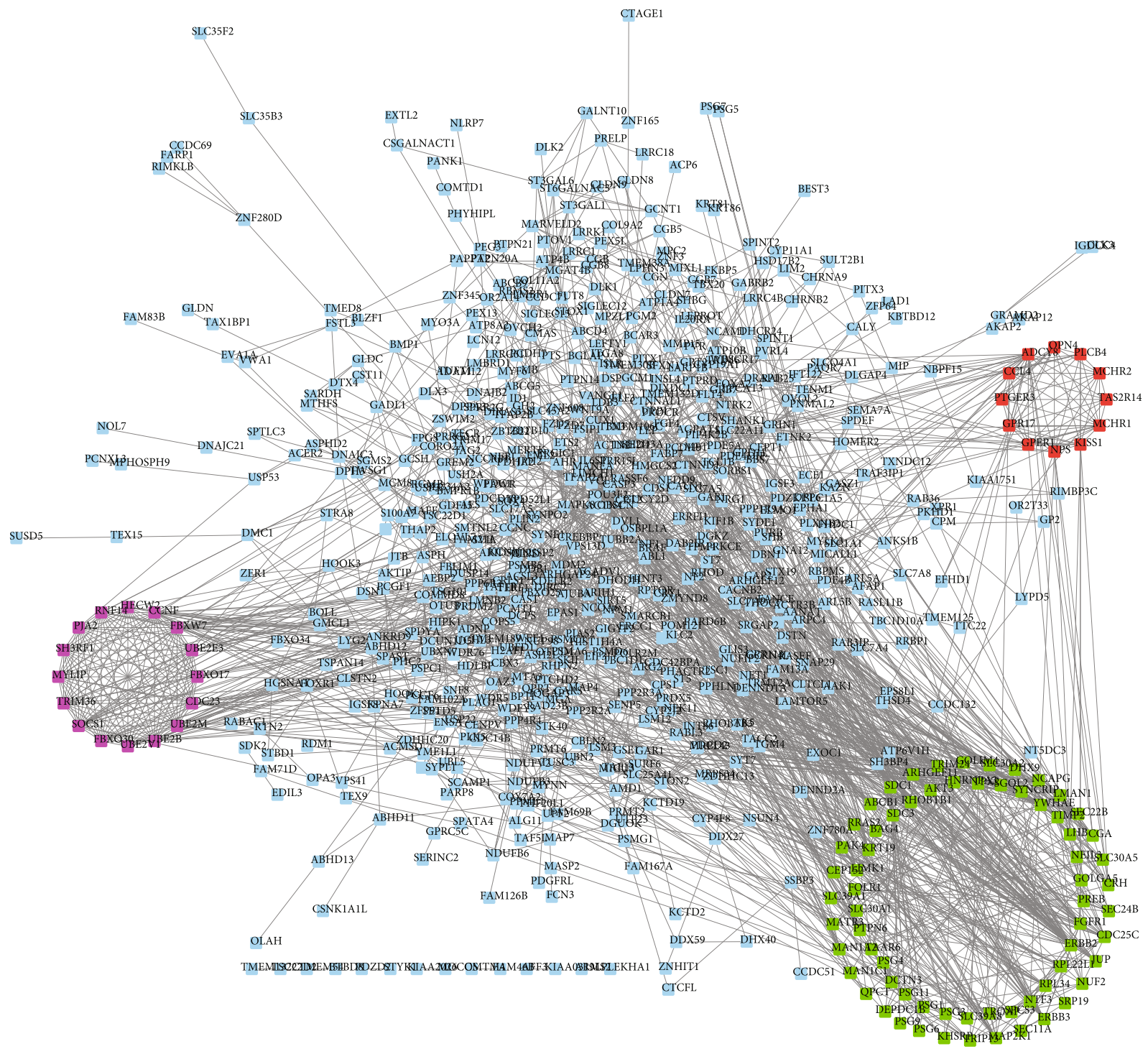

FIGURE 4: Protein-protein interaction network analysis of upregulated hypomethylated genes in PC. We constructed PPI networks of upregulated hypomethylated genes in PC.

differentially methylated [35]. Endothelial cells have been confirmed to be acted as the key inducer to angiogenesis via cell-promoting cell metastasis [36]. Notedly, EPCs (endothelial progenitor cells) functioned importantly in the generation of the postnatal blood vessel and vascular homeostasis [37]. The endothelial dysfunction in PE probably led to the destructive fetoplacental angiogenesis and neovasculogenesis [38]. The decreasing level of some proangiogenic factors in the placenta was observed in the early-stage PE not the late-stage PE [38]. There were more than 2 angiogenesisrelated genes with the reduced level in the early-stage PE after comparison with the late-stage PE or control [39]. Currently, our data revealed that the growth/migration of human umbilical vein endothelial cells was suppressed in the earlystage PE compared to that in the late-stage PE or control, suggesting negative regulation of angiogenesis in $\mathrm{PE}$.

In order to identify the hub genes and networks in PE, we conducted a PPI network analysis. The upregulated hypomethylated PPI network was composed of 380 nodes and
1170 edges, while the downregulated hypermethylated PPI network consisted 380 nodes and 1170 edges. Furthermore, we identified 6 hub networks using MCODE plugin in Cytoscape software. Among these genes, MAPK8, CCNF, CDC23, ABL1, NF1, UBE2E3, CD44, and PIK3R1 were identified as key regulators in PE. MAPK8 belonged to mitogenactivated protein kinase (MAPK) family which is critical for cellular function through regulating numerous signaling pathways [40]. A recent study showed that MAPK8, which is necessary for epithelial-mesenchymal transition, is responsible for regulating transcription [41]. CDC23 is a cell cycle regulator, exhibiting importantly in both initiation and elongation of DNA replication $[42,43]$. Loss of NF1 results in dysregulation of MAPK, PI3K, and other signaling cascades, to promote cell proliferation and to inhibit cell apoptosis. UBE2E3 have a key role in regulation of cell aging which was essential for homeostasis of tissues. Cells' absence of UBE2E3 will be senescent even though without DNA damage [44]; meanwhile, accumulated mitochondrial and lysosomal 


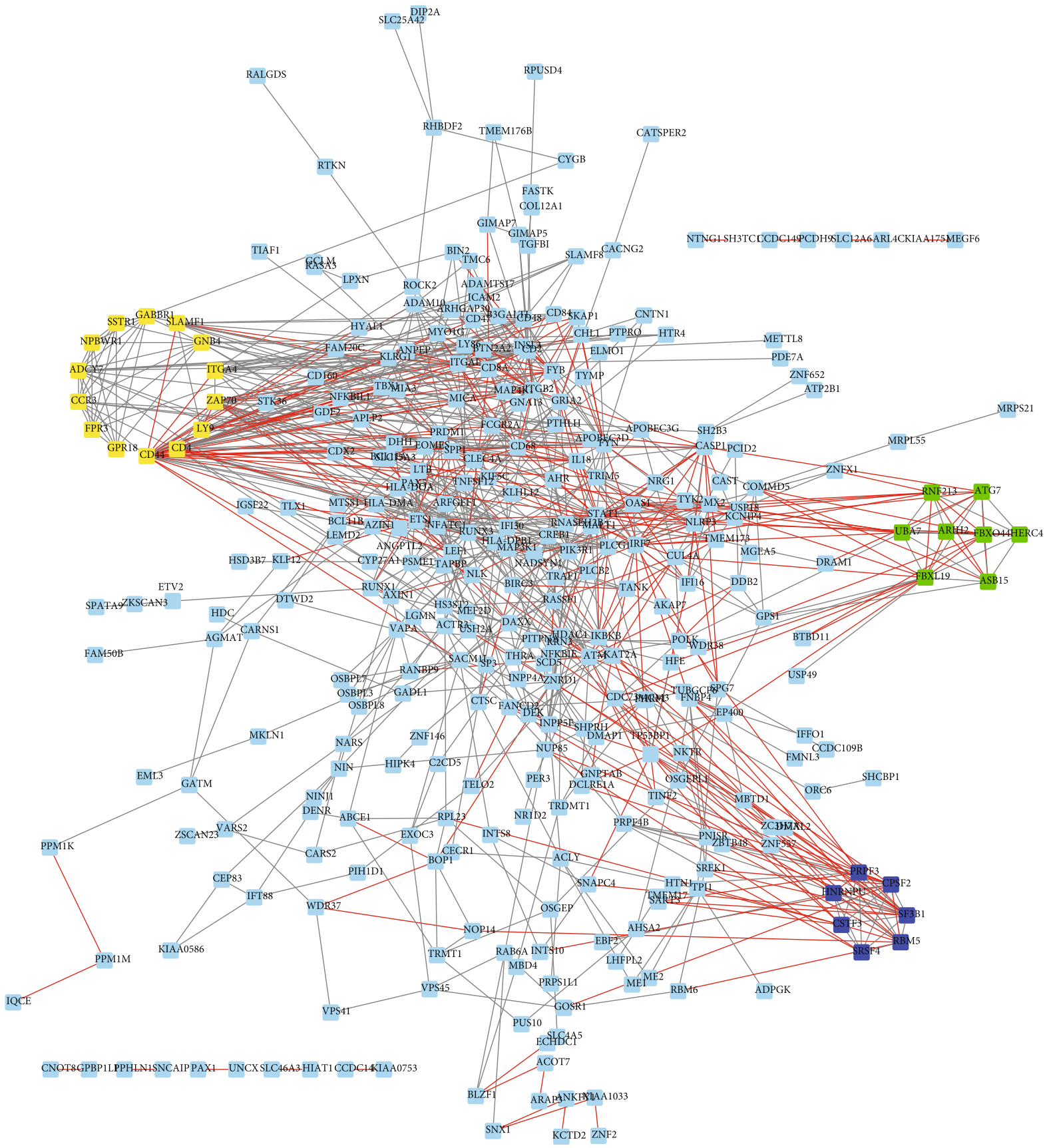

Figure 5: Protein-protein interaction network analysis of downregulated hypermethylated genes in PC. We constructed PPI networks of downregulated hypermethylated genes in PC.

mass and raised basal autophagic flux were shown in UBE2E3 absent cells. CD44 as a member of CAM family mostly takes part in cell movement and proliferation [45]. PIK3R1-encoded PI3K, p85 $\alpha$, could conjugate, maintain, and suppress catalytic subunit of PI3K p110 [46]. Not only did mutated PIK3R1 reduce the subtype of P110 inhibition but also destroyed the new regulatory effect of $\mathrm{p} 85 \alpha$ on PTEN or activated a new signal pathway.

Nevertheless, our studies still had some limitations. Firstly, our researches concentrated on the classification of DEG with different methylations. Secondly, our researches should broaden the analysis datasets so as to acquire com- prehensive data. Thirdly, we needed to conduct qRT-PCR or western blot to further ensure the selected gene level in PE samples. Finally, the function and mechanism of biomarkers in PE need to be further studied in vivo and in vitro.

\section{Conclusion}

Collectively, we identified some oncogene expression patterns and their links with corresponding pathways in PE, providing a hint of exploring the mechanisms implicated in PE onset and development. 


\section{Data Availability}

The datasets used during the present study are available from the corresponding author upon reasonable request.

\section{Conflicts of Interest}

The authors declare that they have no competing interests.

\section{Acknowledgments}

This work has been supported by the Key Discipline Construction of National Health Commission in Pudong New Area of Shanghai Obstetrics and Gynecology (PWZxk201714) and the Establishment and Popularization of Standardized System for Screening and Comprehensive Prevention of Preterm Labor (2019SY044).

\section{References}

[1] T. A. Gudeta and T. M. Regassa, "Pregnancy induced hypertension and associated factors among women attending delivery service at Mizan-Tepi University Teaching Hospital, Tepi General Hospital and Gebretsadik Shawo Hospital, Southwest, Ethiopia," Ethiopian journal of health sciences, vol. 29, no. 1, pp. 831-840, 2019.

[2] M. C. Remich and E. Q. Youngkin, "Factors associated with pregnancy-induced hypertension," The Nurse Practitioner, vol. 14, no. 1, pp. 20-24, 1989.

[3] K. Watanabe, C. Kimura, A. Iwasaki et al., "Pregnancyinduced hypertension is associated with an increase in the prevalence of cardiovascular disease risk factors in Japanese women," Menopause, vol. 22, no. 6, pp. 656-659, 2015.

[4] C. E. Powe, R. J. Levine, and S. A. Karumanchi, "Preeclampsia, a disease of the maternal endothelium: the role of antiangiogenic factors and implications for later cardiovascular disease," Circulation, vol. 123, no. 24, pp. 2856-2869, 2011.

[5] F. Lyall, J. N. Bulmer, E. Duffie, F. Cousins, A. Theriault, and S. C. Robson, "Human trophoblast invasion and spiral artery transformation," The American Journal of Pathology, vol. 158, no. 5, pp. 1713-1721, 2001.

[6] J. Mayrink, M. L. Costa, and J. G. Cecatti, "Preeclampsia in 2018: revisiting concepts, physiopathology, and prediction," Scientific World Journal, vol. 2018, article 6268276, pp. 1-9, 2018.

[7] R. Townsend, P. O'Brien, and A. Khalil, "Current best practice in the management of hypertensive disorders in pregnancy," Integrated Blood Pressure Control, vol. 9, pp. 79-94, 2016.

[8] K. Liu, Q. Fu, Y. Liu, and C. Wang, “An integrative bioinformatics analysis of microarray data for identifying hub genes as diagnostic biomarkers of preeclampsia," Bioscience Reports, vol. 39, no. 9, 2019.

[9] S. Liu, X. Xie, H. Lei, B. Zou, and L. Xie, "Identification of key circRNAs/lncRNAs/miRNAs/mRNAs and pathways in preeclampsia using bioinformatics analysis," Medical Science Monitor, vol. 25, pp. 1679-1693, 2019.

[10] E. Tejera, M. Cruz-Monteagudo, G. Burgos et al., "Consensus strategy in genes prioritization and combined bioinformatics analysis for preeclampsia pathogenesis," BMC Medical Genomics, vol. 10, no. 1, p. 50, 2017.
[11] E. Tejera, J. Bernardes, and I. Rebelo, "Preeclampsia: a bioinformatics approach through protein-protein interaction networks analysis," BMC Systems Biology, vol. 6, no. 1, p. 97, 2012.

[12] H. E. J. Yong, P. E. Melton, M. P. Johnson et al., "Genomewide transcriptome directed pathway analysis of maternal pre-eclampsia susceptibility genes," PLoS One, vol. 10, no. 5, article e0128230, 2015.

[13] M. Trerotola, V. Relli, P. Simeone, and S. Alberti, "Epigenetic inheritance and the missing heritability," Human Genomics, vol. 9, no. 1, p. 17, 2015.

[14] M. Slatkin, "Epigenetic inheritance and the missing heritability problem," Genetics, vol. 182, no. 3, pp. 845-850, 2009.

[15] W. Xu, F. Wang, Z. Yu, and F. Xin, "Epigenetics and cellular metabolism," Genetics \& Epigenetics, vol. 8, pp. 43-51, 2016.

[16] X. Cao and S. E. Jacobsen, "Role of the arabidopsis DRM methyltransferases in de novo DNA methylation and gene silencing," Current Biology, vol. 12, no. 13, pp. 1138-1144, 2002.

[17] S. L. Wilson, K. Leavey, B. J. Cox, and W. P. Robinson, "Mining DNA methylation alterations towards a classification of placental pathologies," Human Molecular Genetics, vol. 27, no. 1, pp. 135-146, 2018.

[18] S. Davis and P. S. Meltzer, "GEOquery: a bridge between the Gene Expression Omnibus (GEO) and BioConductor," Bioinformatics, vol. 23, no. 14, pp. 1846-1847, 2007.

[19] I. Diboun, L. Wernisch, C. A. Orengo, and M. Koltzenburg, "Microarray analysis after RNA amplification can detect pronounced differences in gene expression using limma," $B M C$ Genomics, vol. 7, no. 1, p. 252, 2006.

[20] D. W. Huang, B. T. Sherman, and R. A. Lempicki, "Bioinformatics enrichment tools: paths toward the comprehensive functional analysis of large gene lists," Nucleic Acids Research, vol. 37, no. 1, pp. 1-13, 2009.

[21] G. D. Bader and C. W. Hogue, "An automated method for finding molecular complexes in large protein interaction networks," BMC Bioinformatics, vol. 4, no. 1, p. 2, 2003.

[22] C. H. Chin, S. H. Chen, H. H. Wu, C. W. Ho, M. T. Ko, and C. Y. Lin, "cytoHubba: identifying hub objects and subnetworks from complex interactome," BMC Systems Biology, vol. 8, Supplement 4, p. S11, 2014.

[23] D. B. Nelson, L. F. Chalak, D. D. McIntire, and K. J. Leveno, "Is preeclampsia associated with fetal malformation? A review and report of original research," The Journal of MaternalFetal \& Neonatal Medicine, vol. 28, no. 18, pp. 2135-2140, 2014.

[24] L. Han, Y. Liu, S. Duan, B. Perry, W. Li, and Y. He, "DNA methylation and hypertension: emerging evidence and challenges," Briefings in Functional Genomics, vol. 15, no. 6, pp. 460-469, 2016.

[25] K. A. Pennington, J. M. Schlitt, D. L. Jackson, L. C. Schulz, and D. J. Schust, "Preeclampsia: multiple approaches for a multifactorial disease," Disease Models \& Mechanisms, vol. 5, pp. 9-18, 2011.

[26] R. R. Resende, L. M. Andrade, A. G. Oliveira, E. S. Guimaraes, S. Guatimosim, and M. F. Leite, "Nucleoplasmic calcium signaling and cell proliferation: calcium signaling in the nucleus," Cell Communication and Signaling: CCS, vol. 11, no. 1, p. 14, 2013.

[27] B. W. Poovaiah and L. Du, "Calcium signaling: decoding mechanism of calcium signatures," The New Phytologist, vol. 217, no. 4, pp. 1394-1396, 2018. 
[28] G. Antunes, "Modelling intracellular competition for calcium: kinetic and thermodynamic control of different molecular modes of signal decoding," Scientific Reports, vol. 6, no. 1, p. 23730, 2016.

[29] A. G. Oliveira, E. S. Guimaraes, L. M. Andrade, G. B. Menezes, and M. Fatima Leite, "Decoding calcium signaling across the nucleus," Physiology (Bethesda), vol. 29, no. 5, pp. 361-368, 2014.

[30] P. Maroni, P. Bendinelli, and R. Piccoletti, "Intracellular signal transduction pathways induced by leptin in $\mathrm{C} 2 \mathrm{C} 12$ cells," Cell Biology International, vol. 29, no. 7, pp. 542-550, 2005.

[31] W. E. Muller, D. Ugarkovic, V. Gamulin, B. E. Weiler, and H. C. Schroder, "Intracellular signal transduction pathways in sponges," Electron Microscopy Reviews, vol. 3, no. 1, pp. 97-114, 1990.

[32] F. C. Tsai, G. H. Kuo, S. W. Chang, and P. J. Tsai, "Ca2+ signaling in cytoskeletal reorganization, cell migration, and cancer metastasis," BioMed Research International, vol. 2015, Article ID 409245, 13 pages, 2015.

[33] E. R. Purba, E. I. Saita, and I. N. Maruyama, "Activation of the EGF receptor by ligand binding and oncogenic mutations: the "rotation model"," Cells, vol. 6, no. 2, p. 13, 2017.

[34] G. Ambrosini, J. Plescia, K. C. Chu, K. A. High, and D. C. Altieri, "Activation-dependent exposure of the inter-EGF sequence Leu83-Leu88 in factor Xa mediates ligand binding to effector cell protease receptor-1," The Journal of Biological Chemistry, vol. 272, no. 13, pp. 8340-8345, 1997.

[35] L. Anton, A. G. Brown, M. S. Bartolomei, and M. A. Elovitz, "Differential methylation of genes associated with cell adhesion in preeclamptic placentas," PLoS One, vol. 9, no. 6, article e100148, 2014.

[36] R. Heidenreich, M. Rocken, and K. Ghoreschi, "Angiogenesis drives psoriasis pathogenesis," International Journal of Experimental Pathology, vol. 90, no. 3, pp. 232-248, 2009.

[37] M. Shibuya, "Differential roles of vascular endothelial growth factor receptor-1 and receptor-2 in angiogenesis," Journal of Biochemistry and Molecular Biology, vol. 39, no. 5, pp. 469478, 2006.

[38] C. Escudero, J. M. Roberts, L. Myatt, and I. Feoktistov, "Impaired adenosine-mediated angiogenesis in preeclampsia: potential implications for fetal programming," Frontiers in Pharmacology, vol. 5, p. 134, 2014.

[39] K. Junus, M. Centlow, A. K. Wikstrom, I. Larsson, S. R. Hansson, and M. Olovsson, "Gene expression profiling of placentae from women with early- and late-onset pre-eclampsia: downregulation of the angiogenesis-related genes ACVRL1 and EGFL7 in early-onset disease," Molecular Human Reproduction, vol. 18, no. 3, pp. 146-155, 2012.

[40] M. Cargnello and P. P. Roux, "Activation and function of the MAPKs and their substrates, the MAPK-activated protein kinases," Microbiology and Molecular Biology Reviews, vol. 75, no. 1, pp. 50-83, 2011.

[41] N. Tiwari, N. Meyer-Schaller, P. Arnold et al., "Klf4 is a transcriptional regulator of genes critical for EMT, including Jnk1 (Mapk8)," PLoS One, vol. 8, no. 2, article e57329, 2013.

[42] B. Singh, K. K. Bisht, U. Upadhyay et al., "Role of $\mathrm{Cdc} 23 / \mathrm{Mcm} 10$ in generating the ribonucleotide imprint at the mat1 locus in fission yeast," Nucleic Acids Research, vol. 47, no. 7, pp. 3422-3433, 2019.

[43] S. Prinz, E. S. Hwang, R. Visintin, and A. Amon, "The regulation of Cdc20 proteolysis reveals a role for APC components
Cdc23 and Cdc27 during S phase and early mitosis," Current Biology, vol. 8, no. 13, pp. 750-760, 1998.

[44] K. S. Plafker, K. Zyla, W. Berry, and S. M. Plafker, "Loss of the ubiquitin conjugating enzyme UBE2E3 induces cellular senescence," Redox Biology, vol. 17, pp. 411-422, 2018.

[45] A. Ouhtit, B. Rizeq, H. A. Saleh, M. D. M. Rahman, and H. Zayed, "Novel CD44-downstream signaling pathways mediating breast tumor invasion," International Journal of Biological Sciences, vol. 14, no. 13, pp. 1782-1790, 2018.

[46] L. M. Thorpe, J. M. Spangle, C. E. Ohlson et al., "PI3K-p110 $\alpha$ mediates the oncogenic activity induced by loss of the novel tumor suppressor PI3K-p85 $\alpha$," Proceedings of the National Academy of Sciences of the United States of America, vol. 114, no. 27, pp. 7095-7100, 2017. 